BIOLOGICAL CRYSTALLOGRAPHY

ISSN 1399-0047

Received 4 March 2015

Accepted 5 July 2015

Edited by Q. Hao, University of Hong Kong

Keywords: NO66; complex structure; catalytic mechanism; substrate recognition.

PDB references: $\mathrm{NO}_{6}{ }^{176-\mathrm{C}}-\mathrm{Ni}^{2+}-\mathrm{NOG}$, 4y33; NO66 ${ }^{176-\mathrm{C}}-\mathrm{Ni}^{2+}-\mathrm{NOG}-\mathrm{RPL} 8^{204-224}$, 4y3o; NO66, M2 mutant, 4y4r

Supporting information: this article has supporting information at journals.iucr.org/d

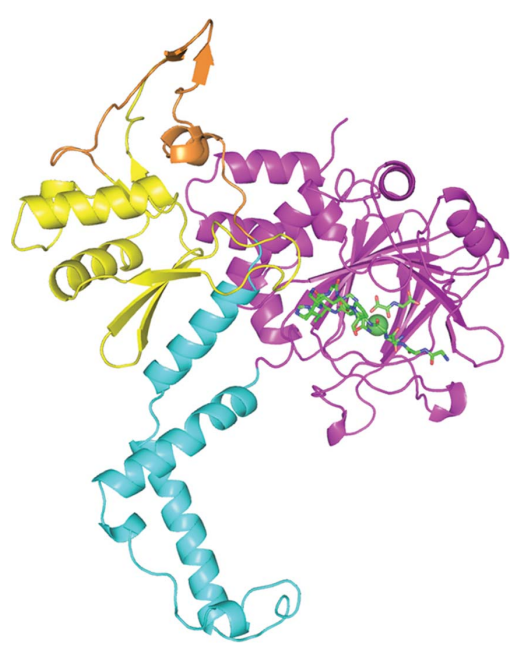

OPEN $\odot$ ACCESS

\section{Structure of the JmjC domain-containing protein NO66 complexed with ribosomal protein Rpl8}

\author{
Chengliang Wang, ${ }^{a, b}$ Qiongdi Zhang, ${ }^{a}$ Tianrong Hang, ${ }^{a, b}$ Yue Tao, ${ }^{c}$ Xukai Ma, \\ Minhao $\mathrm{Wu}^{\mathrm{a}}{ }^{\mathrm{a}, \mathrm{b}}$ Xuan Zhang ${ }^{\mathrm{a}, \mathrm{b} *}$ and Jianye Zang ${ }^{\mathrm{a}, \mathrm{b} *}$
}

\begin{abstract}
a Hefei National Laboratory for Physical Sciences at Microscale and School of Life Sciences, Collaborative Innovation Center of Chemistry for Life Science, University of Science and Technology of China, 96 Jinzhai Road, Hefei, Anhui 230026, People's Republic of China, '⿳亠丷厂犬ney Laboratory of Structural Biology, Chinese Academy of Sciences, Hefei, Anhui 230026, People's Republic of China, and 'Institute for Pediatric Translational Medicine, Shanghai Children's Medical Center, 1678 Dongfang Road, Pudong, Shanghai 200120, People's Republic of China. *Correspondence e-mail: xuanzbin@ustc.edu.cn, zangjy@ustc.edu.cn
\end{abstract}

The JmjC domain-containing proteins belong to a large family of oxygenases possessing distinct substrate specificities which are involved in the regulation of different biological processes, such as gene transcription, RNA processing and translation. Nucleolar protein 66 (NO66) is a JmjC domain-containing protein which has been reported to be a histone demethylase and a ribosome protein 8 (Rp18) hydroxylase. The present biochemical study confirmed the hydroxylase activity of NO66 and showed that oligomerization is required for NO66 to efficiently catalyze the hydroxylation of Rpl8. The structures of NO66 ${ }^{176-C}$ complexed with $\mathrm{Rpl}^{204-224}$ in a tetrameric form and of the mutant protein M2 in a dimeric form were solved. Based on the results of structural and biochemical analyses, the consensus sequence motif $\mathrm{NH} X \mathrm{H}$ recognized by NO66 was confirmed. Several potential substrates of NO66 were found by a BLAST search according to the consensus sequence motif. When binding to substrate, the relative positions of each subunit in the NO66 tetramer shift. Oligomerization may facilitate the motion of each subunit in the NO66 tetramer and affect the catalytic activity.

\section{Introduction}

The nonhaem $\alpha$-ketoglutarate $(\alpha \mathrm{KG}) / \mathrm{Fe}^{2+}$-dependent oxygenases were originally identified as prolyl and lysyl hydroxylases that have essential roles in collagen biosynthesis (Myllyharju \& Kivirikko, 2004; McDonough et al., 2010). Subsequently, $\alpha \mathrm{KG} / \mathrm{Fe}^{2+}$-dependent oxygenases were found to be widely distributed in most organisms and were recognized as a superfamily of enzymes catalyzing various oxidation reactions. These enzymes bear similar catalytic domains folded into double-stranded $\beta$-helical (DSBH) structures with a conserved His- $X$-Glu/Asp- $X_{n}$-His motif responsible for the coordination of $\mathrm{Fe}^{2+}$ (Que, 2000; Clifton et al., 2006). Other motifs or domains surrounding the catalytic core structure further differentiate these enzymes into subfamilies. These domains may be involved in the regulation of enzymatic activity, thus acting to determine the function of the individual enzymes (McDonough et al., 2010).

The JmjC domain-containing proteins comprise a subclass of $\alpha \mathrm{KG} / \mathrm{Fe}^{2+}$-dependent oxygenases which possess distinct substrate specificities and participate in various cellular processes, including regulation of gene expression (Chen et al., 2006; Whetstine et al., 2006), mRNA splicing (Webby et al., 
Table 1

Data-collection and refinement statistics.

Values in parentheses are for the highest resolution shell.

\begin{tabular}{|c|c|c|}
\hline & $\begin{array}{l}\text { NO66 }^{176-\mathrm{C}}-\mathrm{Ni}^{2+}- \\
\text { NOG-Rpl } 8^{204-224}\end{array}$ & M2 \\
\hline \multicolumn{3}{|l|}{ Data collection } \\
\hline Space group & $C 2$ & $P 2_{1} 2_{1} 2_{1}$ \\
\hline \multicolumn{3}{|l|}{ Unit-cell parameters } \\
\hline$a(\AA)$ & 88.50 & 86.06 \\
\hline$b(\AA)$ & 202.93 & 144.21 \\
\hline$c(\AA)$ & 85.68 & 144.21 \\
\hline$\alpha=\gamma\left({ }^{\circ}\right)$ & 90 & 90 \\
\hline$\beta\left({ }^{\circ}\right)$ & 118.94 & 90 \\
\hline Resolution $(\AA)$ & $\begin{array}{l}50.0-2.2 \\
\quad(2.32-2.20)\end{array}$ & $\begin{array}{l}50.0-3.3 \\
\quad(3.48-3.30)\end{array}$ \\
\hline No. of unique reflections & 62029 & 27284 \\
\hline Wilson plot $B$ factor $\left(\AA^{2}\right)$ & 20.0 & 73.7 \\
\hline$R_{\text {meas }} \dagger(\%)$ & $14.6(59.7)$ & $11.0(67.6)$ \\
\hline Mean $I / \sigma(I)$ & $7.6(2.4)$ & $12.4(3.0)$ \\
\hline Completeness (\%) & $98.0(97.8)$ & $98.8(98.0)$ \\
\hline Multiplicity & $3.4(3.4)$ & $6.2(5.6)$ \\
\hline \multicolumn{3}{|l|}{ Refinement } \\
\hline Resolution (Å) & $50.0-2.2$ & $50.0-3.3$ \\
\hline$R_{\text {work }} \ddagger / R_{\text {free }} \S(\%)$ & $20.43 / 26.03$ & $21.81 / 27.21$ \\
\hline \multicolumn{3}{|l|}{ No. of atoms } \\
\hline Protein & 7535 & 7076 \\
\hline Water & 561 & 4 \\
\hline Other ligand & 22 & 6 \\
\hline \multicolumn{3}{|l|}{ R.m.s. deviations } \\
\hline Bond lengths $(\AA)$ & 0.014 & 0.0142 \\
\hline Bond angles $\left({ }^{\circ}\right)$ & 1.559 & 1.641 \\
\hline \multicolumn{3}{|l|}{$B$ factors $\left(\AA^{2}\right)$} \\
\hline Protein & 24.47 & 100.35 \\
\hline Water & 28.56 & 61.25 \\
\hline Other ligand & 30.67 & 57.67 \\
\hline \multicolumn{3}{|l|}{ Ramachandran plot } \\
\hline Most favoured regions (\%) & 96.29 & 92.73 \\
\hline Additionally allowed regions (\%) & 3.71 & 7.27 \\
\hline Outliers $(\%)$ & 0 & 0 \\
\hline
\end{tabular}

$\dagger R_{\text {meas }}$ was estimated by multiplying the conventional $R_{\text {merge }}$ value by the factor $[N /(N-$ $1)]^{1 / 2}$, where $N$ is the data multiplicity; $R_{\text {merge }}=\sum_{h k l} \sum_{i}\left|I_{i}(h k l)-\langle I(h k l)\rangle\right| /$ $\sum_{h k l} \sum_{i} I_{i}(h k l)$, where $I_{i}(h k l)$ is the intensity of the $i$ th measurement and $\langle I(h k l)\rangle$ is the mean intensity for that reflection. $\ddagger R_{\text {work }}=\sum_{h k l}|| F_{\text {obs }}|-| F_{\text {calc }}|| / \sum_{h k l}\left|F_{\text {obs }}\right|$, where $\left|F_{\text {obs }}\right|$ and $\left|F_{\text {calc }}\right|$ are the observed and calculated structure-factor amplitudes, respectively. $\quad \S R_{\text {free }}$ was calculated as for $R_{\text {work }}$ but using the $5.0 \%$ of the reflections in the test set.

2009) and tRNA modification (Kato et al., 2011). A large subfamily of JmjC domain-containing proteins function as histone demethylases, which participate in many fundamental biological processes such as transcriptional regulation and epigenetic inheritance (Jenuwein \& Allis, 2001; Martin \& Zhang, 2005). Other JmjC domain-containing proteins catalyze the oxidation of nonhistone proteins and nucleic acids, and have been shown to be essential for the regulation of gene expression, RNA processing and the fidelity of translation (Chowdhury et al., 2009; Webby et al., 2009; Kato et al., 2011).

Nucleolar protein 66 (NO66) is a metazoan JmjC domaincontaining protein that is highly conserved throughout evolution and was initially identified as a dual-location intranuclear protein (Eilbracht et al., 2004). In the nucleus, NO66 was reported to interact with the osteoblast-specific transcription factor osterix (Osx) and to regulate the expression of Osx-dependent genes and bone formation (Sinha et al., 2010). In addition, recent work showed that NO66 is recruited to the stem-cell genes together with the PRC2 (polycomb repressor complexes 2) complex during differentiation, resulting in gene silencing (Brien et al., 2012). In both cases, NO66 was reported to be a histone demethylase in vivo. Evidence from a recent study indicated that NO66 hydroxylates ribosome protein 8 ( Rpl8) and controls the biogenesis of the ribosome (Ge et al., 2012), which is consistent with the observation that NO66 is a constitutive nucleolar component (Eilbracht et al., 2004). Moreover, previous work demonstrated that NO66 assembles as a tetramer and that the binding site of NO66 to osterix is located on the surface formed by a hinge region (interface I) from neighbouring subunits in the tetramer (Tao et al., 2013). Although tetramer formation has been shown to be important for the interaction of NO66 with osterix and the regulation of gene transcription, whether tetrameric assembly is required for its catalytic activity remains unknown. Thus, further investigation of the enzymatic activity of NO66 is required. While this manuscript was under preparation, Chowdhury and coworkers published the structure of NO66 in complex with an Rpl8 peptide using a disulfide-based cross-linking strategy, suggesting a potential interaction between NO66 and Rpl8 (Chowdhury et al., 2014). However, further research is required to understand the mechanism of interaction between NO66 and Rpl8 in the native state.

Here, we performed biochemical assays to confirm that NO66 hydroxylates Rpl8. In our assays, oligomerization is required for NO66 to hydroxylate Rpl8 efficiently. In addition, we determined the structures of NO66 ${ }^{176-\mathrm{C}}$ complexed with Rpl $8^{204-224}$ and the mutant protein M2 lacking one of the dimerization interfaces in NO66. Based on structural and biochemical analyses, we confirmed that NO66 recognizes a consensus sequence motif and found that the relative positions of each subunit in the NO66 tetramer change upon substrate binding. Based on these observations, we proposed that oligomerization could affect the catalytic activity of NO66.

\section{Methods}

\subsection{Protein purification and crystallization}

The C-terminal fragment of wild-type human NO66 (NO66 ${ }^{176-\mathrm{C}}$ ) was cloned, expressed and purified as described previously (Zhou et al., 2012; Tao et al., 2013). In brief, NO66 $^{176-\mathrm{C}}$ or its mutants were cloned into pET-28a(+) (Novagen) with a His-SUMO tag fused at the N-terminus and were expressed in Escherichia coli Rosetta 2 (DE3) cells. The target proteins were purified using Ni-NTA resin (GE Healthcare). The His-SUMO tag was removed by TEV protease and the proteins were further purified using a HiLoad 10/300 Superdex 200 column (GE Healthcare) in buffer $A$ (20 m $M$ Tris-HCl pH 7.5, $200 \mathrm{~m} M \mathrm{NaCl})$. The eluted fractions corresponding to target proteins were collected and concentrated for further use.

Crystals of NO66 ${ }^{176-C}$ in complex with $\mathrm{Rp}^{204-224}$ peptide (human ribosomal protein L8) were obtained using the sittingdrop vapour-diffusion method at $295 \mathrm{~K}$. Before crystallization, $\mathrm{Ni}^{2+}$, NOG ( $N$-oxalylglycine, an analogue of $\alpha$-ketoglutarate) and Rpl8 peptide were added to the protein solution with a 
final molar ratio of 1:4:10:15 (protein:NOG: $\mathrm{Ni}^{2+}$ :peptide) and the mixture was incubated for about $1 \mathrm{~h}$. The crystal used for data collection was grown in a solution consisting of $0.1 \mathrm{M}$ imidazole $\mathrm{pH}$ 6.5, $0.5 \mathrm{M}$ sodium acetate trihydrate. Crystals of the M2 mutant in complex with $\mathrm{Ni}^{2+}$ were grown using the same procedures. The final crystallization condition for M2 was $1.0 \mathrm{M}\left(\mathrm{NH}_{4}\right)_{2} \mathrm{HPO}_{4}, 0.1 \mathrm{M}$ acetate $\mathrm{pH} 4.5$.

\subsection{Data collection and structure determination}

The crystals were gradually transferred into cryoprotectant solution supplemented with $20 \%(v / v)$ glycerol and flashcooled in liquid nitrogen. X-ray diffraction data were collected at $100 \mathrm{~K}$ on beamline BL17U1 at the Shanghai Synchrotron Radiation Facility. Diffraction data were indexed, integrated and scaled using iMosflm (Battye et al., 2011) and POINTLESS and SCALA (Evans, 2006) from the CCP4 suite (Winn et al., 2011). The structure of NO66 ${ }^{176-\mathrm{C}}$ in complex with Rpl8 peptide was solved by molecular replacement (McCoy et al., 2007) using the structure of NO66 ${ }^{183-C}$ (PDB entry $4 \mathrm{e} 4 \mathrm{~h} ; \mathrm{Y}$. Tao, M. Wu, K. M. Sinha \& J. Zang, unpublished work) as a search model. The structure was refined to $2.2 \AA$ resolution

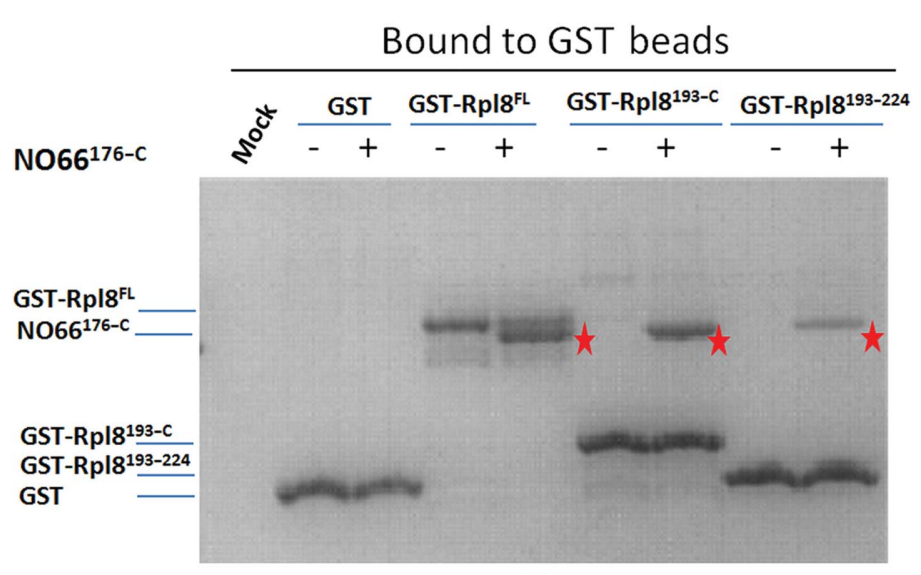

(a)

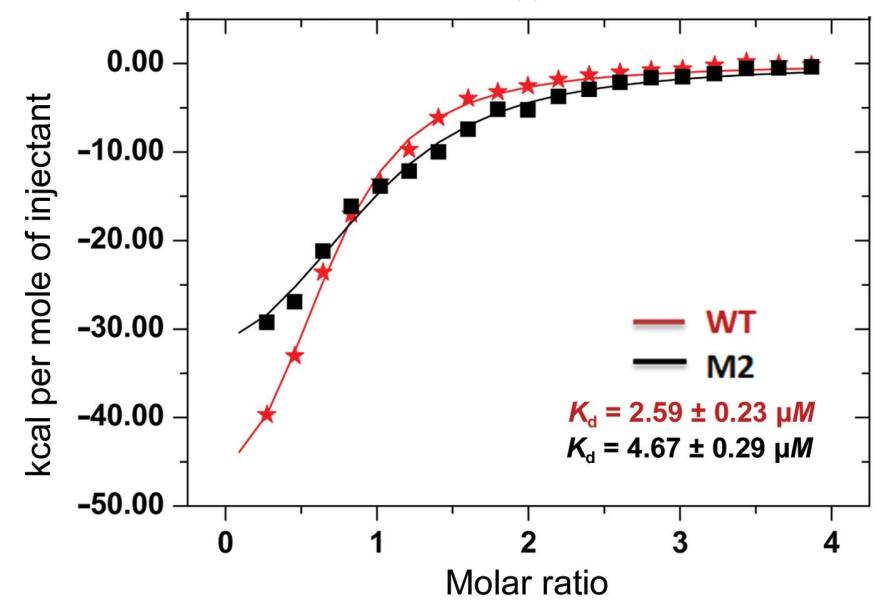

(c)

Figure 1 using REFMAC5 (Murshudov et al., 2011) and Coot (Emsley \& Cowtan, 2004). Similar methods were used to refine the structure of the M2 mutant. The final structural models were validated using PROCHECK (Laskowski et al., 1993). The data-processing and structure-determination statistics are listed in Table 1. All of the structural figures were prepared using PyMOL (http://www.pymol.org).

\subsection{GST pull-down assay}

Various fragments of human Rpl8 were cloned into the pGEX-6P-1 vector and overexpressed in E. coli BL21 (DE3) cells. The cells were lysed in $1 \mathrm{ml}$ buffer $B(50 \mathrm{~m} M$ HEPES $\mathrm{pH}$ 7.5, $300 \mathrm{~m} M \mathrm{NaCl}, 0.1 \%$ Triton $\mathrm{X}-100)$. The lysates were incubated with $20 \mu \mathrm{l}$ GST beads pre-equilibrated in buffer $B$. After washing three times, GST-Rpl8 fragments immobilized with GST beads were incubated with purified NO66 ${ }^{176-\mathrm{C}}$ or mutants at $277 \mathrm{~K}$ for $1 \mathrm{~h}$. The beads were washed with buffer $B$ four times and boiled with SDS sample buffer. The proteins retained on the glutathione Sepharose beads were analyzed using SDS-PAGE.

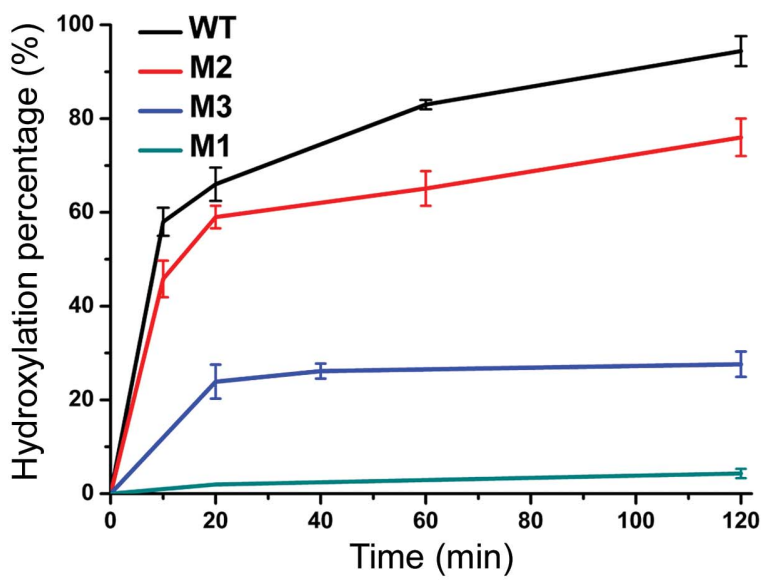

(b)

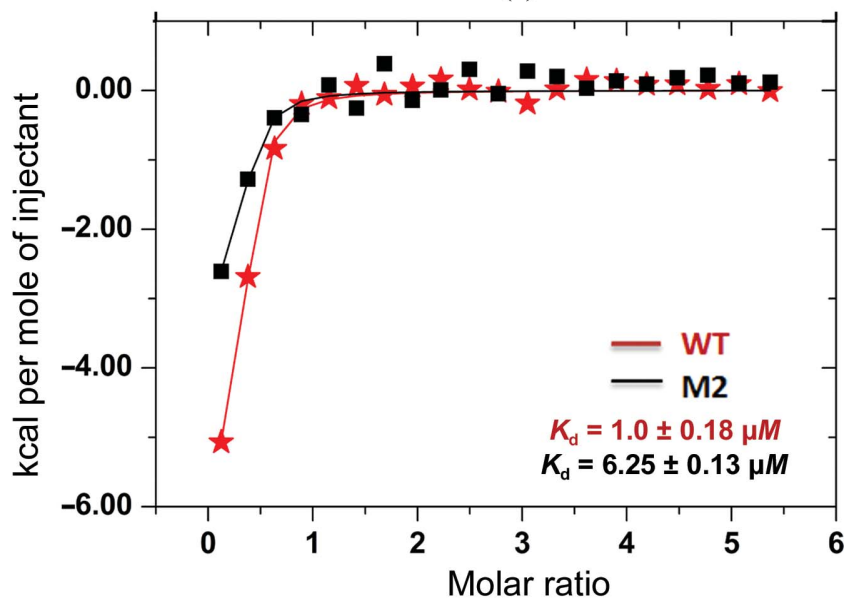

(d)

Oligomerization of NO66 is required for substrate binding and catalysis. (a) GST pull-down of NO66 ${ }^{176-\mathrm{C}}$ by different fragments of Rpl8 fused to GST. Asterisks indicate the band corresponding to NO66 ${ }^{176-C}$. (b) A comparison of the efficiency of wild-type NO66 and mutants. The products of the hydroxylation reaction were detected by LC-MS/MS and the data are semi-quantitative. $(c, d)$ Comparison of the binding of wild-type NO66 ${ }^{176-\mathrm{C}}$ (red) and mutant protein M2 (black) to GST-Rpl8 ${ }^{193-\mathrm{C}}(c)$ and $\alpha \mathrm{KG}(d)$. The ITC method was used to analyze the binding affinities. 


\subsection{Hydroxylation activity analysis}

NO66 ${ }^{176-\mathrm{C}}(10 \mu M)$ was incubated with $\mathrm{Rp} 18^{204-224}$ peptide $(100 \mu M)$ in $100 \mu \mathrm{l}$ buffer $C$ [50 mM HEPES pH 7.5, $300 \mathrm{~m} M$ $\mathrm{NaCl}, 0.5 \mathrm{~m} M$ TCEP, $200 \mu M \alpha \mathrm{KG}, 100 \mu M \mathrm{Fe}\left(\mathrm{NH}_{4}\right)_{2}\left(\mathrm{SO}_{4}\right)_{2}$, $200 \mu M$ ascorbic acid] at room temperature for $60 \mathrm{~min}(\mathrm{Ge}$ et $a l ., 2012)$. The reaction was quenched by the addition of $10 \mu \mathrm{l}$ $0.1 \%$ TFA (trifluoroacetic acid) and the insoluble material was removed by centrifugation. The supernatant was desalted using $\mathrm{C}_{18}$ ZipTips (Millipore; Chen et al., 2006). The bound material was eluted from the $\mathrm{C}_{18}$ ZipTips with $70 \%$ acetonitrile/ $0.1 \%$ TFA with or without $\alpha$-cyano-4-hydroxycinnamic acid. The eluted samples were used for MALDI-TOF MS or LC-MS/MS analysis.

\subsection{Size-exclusion chromatographic analysis}

The purified NO66 $6^{176-\mathrm{C}}$ or mutant (M1, M2 and M3) proteins were loaded onto a HiLoad 10/300 Superdex 200 column (GE Healthcare) pre-equilibrated with the column buffer $(20 \mathrm{~m} M$ Tris- $\mathrm{HCl} \mathrm{pH} 7.5,200 \mathrm{mM} \mathrm{NaCl})$ and eluted using the same buffer. The molecular weight of each protein was estimated according to the elution volume.

\subsection{Isothermal titration calorimetry (ITC)}

ITC measurements were performed at $287 \mathrm{~K}$ as reported previously (Ruan et al., 2012). $40 \mu \mathrm{l} \alpha \mathrm{KG}$ (500 $\mu M$ stock solution) was injected into a sample cell containing $20 \mu M$ wild-type NO66 or mutant protein fused to the SUMO tag in buffer $D(50 \mathrm{~m} M$ Tris- $\mathrm{HCl}$ pH 7.5, $300 \mathrm{~m} M \mathrm{NaCl}, 20 \mu M$ $\mathrm{NiCl}_{2}$ ). To measure the affinity of substrate binding, $40 \mu \mathrm{l}$ GST-Rpl8 ${ }^{193-\mathrm{C}}(270 \mu M$ stock solution) was injected into a sample cell containing $15 \mu M$ protein in buffer $D$ supplemented with $60 \mu M \alpha \mathrm{KG}$. The ITC measurements were fitted to a one-site binding model using the Origin software (MicroCal Inc.).

\section{Results}

3.1. Oligomerization of NO66 is required for substrate binding and catalytic activity

NO66 was recently identified as a protein hydroxylase that is capable of catalyzing histidyl hydroxylation of Rpl8 (Ge et al., 2012). To confirm the activity of NO66, we evaluated the interaction between NO66 and Rpl8. We performed in vitro pull-down assays with several fragments of Rpl8 fused to GST. As expected, the C-terminal fragment of Rpl8 binds to NO66 ${ }^{176-C}$ (Fig. 1a). In addition, a short region of $\mathrm{Rpl} 8$ spanning amino-acid residues 193-224 is sufficient to interact

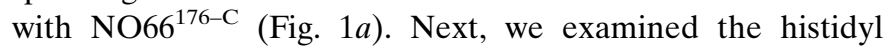
hydroxylation activity of NO66 $6^{176-\mathrm{C}}$ by using a synthetic peptide containing 21 amino-acid residues of Rpl8 (204-224; referred to as $\mathrm{Rpl}^{204-224}$ ) as the substrate. After incubation with $\mathrm{NO}^{176-\mathrm{C}}$, the molecular weight of $\mathrm{Rp} 18^{204-224}$ had a $+16 \mathrm{Da}$ shift, indicating the possible occurrence of hydroxylation (Supplementary Fig. S1a). Further analyses showed that His 216 of Rpl8 is hydroxylated by NO66 ${ }^{176-\mathrm{C}}$ (Supple- mentary Fig. S1b). Our activity assays confirmed that NO66 is a protein histidyl hydroxylase.

Our previous analyses demonstrated that NO66 ${ }^{176-\mathrm{C}}$ exists as a tetramer in solution (Tao et al., 2013). Oligomerization is necessary for NO66 to associate with Osx and this represses the expression of Osx-dependent genes (Tao et al., 2013). However, whether tetramer formation is also necessary for the catalytic activity of NO66 remains unknown. Therefore, we generated several mutants to disrupt the oligomerization of NO66 and compared their catalytic activities with that of the wild-type protein. As reported previously, we had already produced two mutant proteins, M1 and M2, lacking dimer interfaces I and II (Supplementary Fig. S2), and they exist as a monomer and a dimer, respectively (Tao et al., 2013). Because the tetramer of NO66 is formed through dimer interfaces I and II, disruption of these two dimer interfaces would lead to two types of dimers (Supplementary Fig. S2). Unexpectedly, we only obtained monomeric NO66 on the deletion of dimer interface I. Therefore, we produced a third mutant, M3, by mutating three amino-acid residues (F450A/R452A/P455A) in dimer interface $\mathrm{I}$, and this mutant forms a dimer in solution (Supplementary Fig. S2).

After purification of the NO66 mutants, we measured their hydroxylation activity in vitro. The M1 mutant lost catalytic activity. Compared with wild-type NO66, the activity of the M2 protein decreased and the activity of the M3 protein decreased even further (Fig. 1b). These results indicate that oligomerization is necessary for catalytic activity of NO66, and that the dimer interface I of NO66 plays a more important role in catalysis than dimer interface II. In addition, we used ITC assays to measure the binding of wild-type and mutant NO66 to its substrate GST-Rpl $8^{193-\mathrm{C}}$ and cofactor $\alpha \mathrm{KG}$. The binding of wild-type NO66 to GST-Rpl $8^{193-\mathrm{C}}$ is the strongest $\left(K_{\mathrm{d}}=2.59\right.$ $\pm 0.23 \mu M)$. The M2 protein binds to GST-Rpl $8^{193-\mathrm{C}}$, but with a nearly twofold weaker affinity $\left(K_{\mathrm{d}}=4.67 \pm 0.29 \mu M\right)$. The interaction between GST-Rpl ${ }^{193-C}$ and the M1 or M3 mutants may be very weak and may not be detected by ITC assays (Fig. $1 c$ and Supplemetary Fig. S3a). We also investigated the binding of these proteins to the cofactor $\alpha \mathrm{KG}$. Similarly, the binding affinity of wild-type NO66 to $\alpha \mathrm{KG}$ is the highest $\left(K_{\mathrm{d}}=\right.$ $1.0 \pm 0.18 \mu M)$. The M2 mutant binds to $\alpha \mathrm{KG}$ with an approximately sixfold lower affinity $\left(K_{\mathrm{d}}=6.25 \pm 0.13 \mu M\right)$. The binding of the M1 and M3 mutants to $\alpha \mathrm{KG}$ is almost undetectable under these conditions (Fig. $1 d$ and Supplementary Fig. S3b). Collectively, these data suggested that the oligomerization of NO66 is required for substrate and cofactor binding, which may further affect the reaction catalyzed by NO66.

\subsection{Overall structure of NO66 ${ }^{176-C}$ complexed with Rpl8 peptide}

To better understand the catalytic mechanism of NO66, we determined the structure of NO66 in complex with a synthetic peptide derived from Rpl8 (residues 204-224, referred to as Rpl $\left.8^{204-224}\right)$. The fragment of NO66 that we used for structural analysis lacks the $\mathrm{N}$-terminal 175 amino acids (referred to as 
NO66 ${ }^{176-\mathrm{C}}$ ). The $\mathrm{Fe}^{2+}$ ion and $\alpha \mathrm{KG}$ are naturally accommodated in the active site of NO66. To determine the complex structure of $\mathrm{NO}^{176-\mathrm{C}}$ and $\mathrm{Rpl}^{204-224}, \mathrm{Fe}^{2+}$ ion and $\alpha \mathrm{KG}$ were substituted by $\mathrm{Ni}^{2+}$ and NOG to prevent the reaction catalyzed by NO66 during crystallization. The statistics of data collection and refinement are shown in Table 1.

Similar to previous reports (Tao et al., 2013), the structure of NO66 ${ }^{176-C}$ consists of four regions: the JmjC domain (residues 176-426), the hinge-domain region (residues 427-510), a $\beta$-hairpin motif (residues 511-547) and the C-terminal wHTH motif (residues 548-641) (Fig. 2a). In the catalytic centre of NO66 ${ }^{176-C}$, electron density corresponding to Rpl8 residues $212-222$ is clearly visible (Figs. $2 a$ and $2 b$ ). There are two molecules in one asymmetric unit that form a dimer through

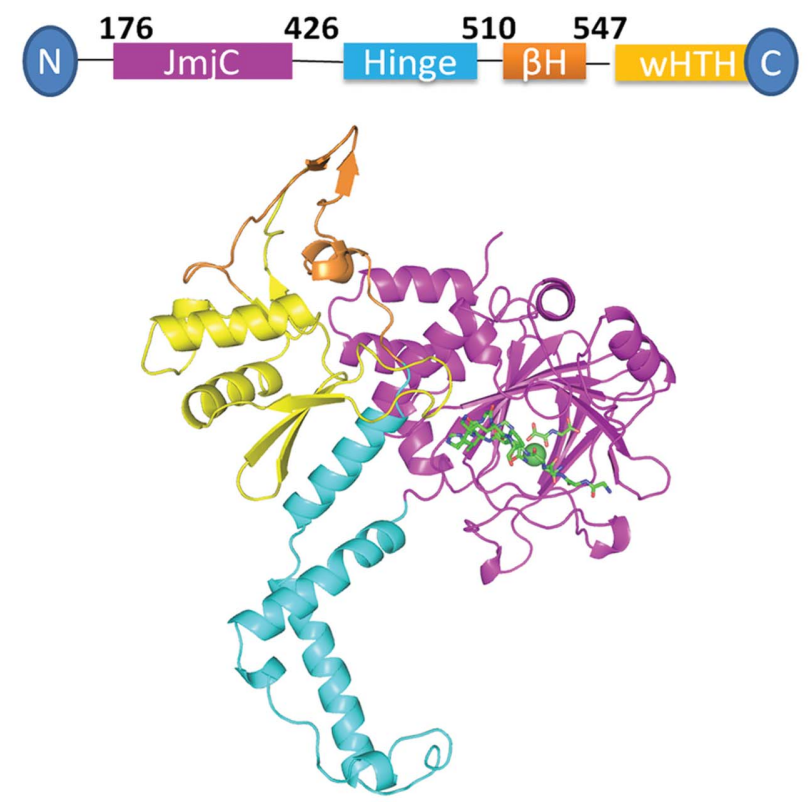

(a)

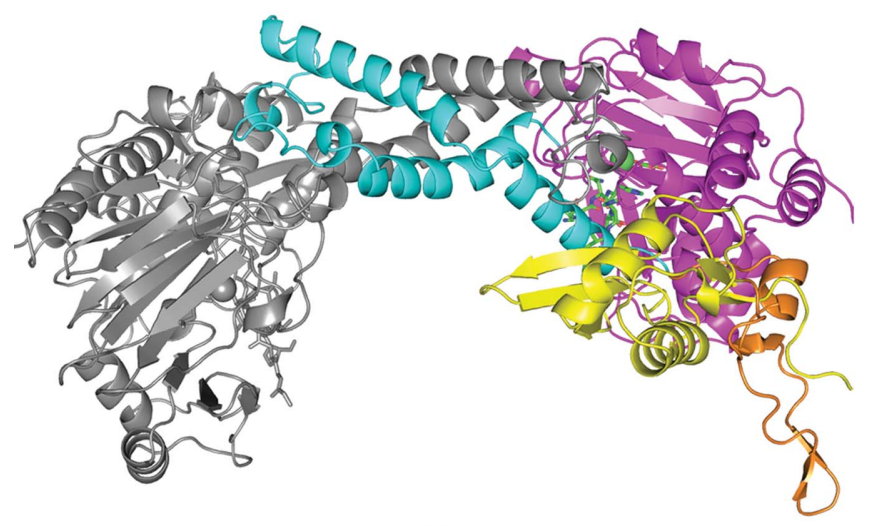

(c) dimer interface I (hinge domain; Fig. 2c). These two molecules are similar to each other, with a main-chain root-mean-square deviation (r.m.s.d.) of $0.22 \AA$. Two neighbouring dimers related by the crystallographic twofold rotation symmetry pack against each other to form the tetramer (Fig. $2 d$ ).

\subsection{Structural basis for specific binding of Rpl8 by NO66}

In the complex structure of NO66 ${ }^{176-\mathrm{C}}$ and $\mathrm{Rpl}^{204-224}$ only residues $212-222$ of Rpl8 are visible in the electron-density map. The other regions of this peptide are invisible, possibly because of high flexibility. From the complex structure, it is apparent that the $\mathrm{Rpl}^{204-224}$ peptide bound to $\mathrm{NO}^{20{ }^{176-\mathrm{C}}}$ in a cleft located in the centre of the JmjC domain (Fig. 3a). The

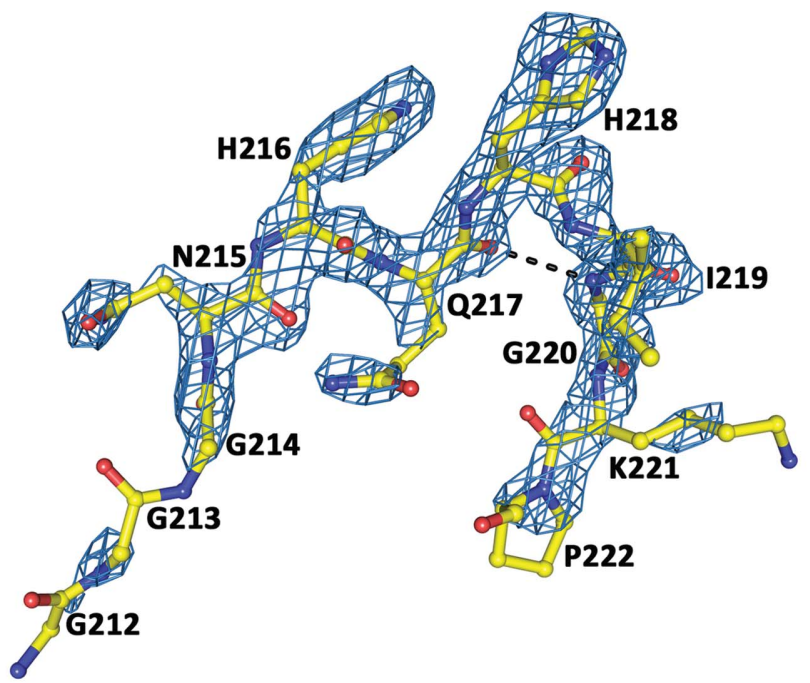

(b)

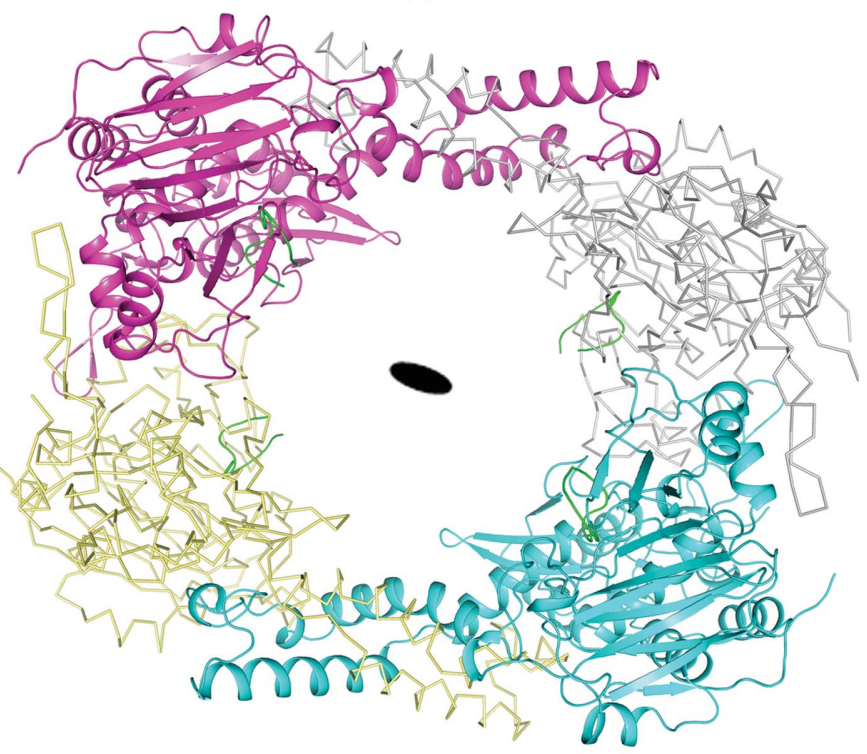

(d)

Figure 2

Overall structure of NO66 ${ }^{176-\mathrm{C}}$ complexed with Rpl8 peptide. (a) Cartoon representation of the crystal structure of NO66 ${ }^{176-\mathrm{C}}$ complexed with $\mathrm{Rpl}^{204-224}$, a peptide derived from Rpl8. The JmjC domain, the dimerization hinge domain, the $\beta$-hairpin motif and the C-terminal wHTH domain are shown in pink, cyan, orange and magenta, respectively. The Rpl8 peptide is coloured green. (b) The bound peptide Rpl8 $8^{204-224}$ is shown as sticks. The $F_{\mathrm{o}}-F_{\mathrm{c}}$ OMIT electron-density map for bound substrate was contoured at 3.0 $\sigma$. A cartoon representation of the dimeric $(c)$ and tetrameric $(d)$ structure of NO66 $6^{176-\mathrm{C}}$ in complex with Rpl $8^{204-224}$ is shown. A dimer is present in the asymmetric unit. Two dimers related by crystallographic twofold symmetry pack against each other to form the tetramer. The filled oval indicates the twofold symmetry axis. 
overall surface area of NO66 covered by the bound peptide is about $659 \AA^{2}$. The Rpl8 $8^{204-224}$ peptide adopts a U-shaped conformation and penetrates deeply into a cleft (Fig. 3a). Residue Gln217 points to the outside of the binding pocket, leading to a bulge shape formed in the middle of the U-shaped $\mathrm{Rpl}^{204-224}$ peptide. This bulge-shaped structure is stabilized by a hydrogen bond formed between the backbone carbonyl group of Gly217 and the backbone amide group of Gly220 of Rpl8 ${ }^{204-224}$ (Fig. 2b).

By analysis of the complex structure of $\mathrm{NO}^{176-\mathrm{C}}$ and $\mathrm{Rp} 18^{204-224}$, we found that the $\mathrm{Rpl} 8^{204-224}$ peptide is tightly embedded in the binding cleft, especially near the four residues (Asn215, His216, Gln217 and His218) located in the middle of the peptide. The $\mathrm{N}$-terminal three glycine residues are on the surface of the entrance to the binding cleft. The backbone carbonyl groups of these glycine residues form hydrogen bonds to the side chains of Arg272, Thr274 and Asn376 of NO66 (Fig. 3b). Residue Asn215 of the Rpl8 $8^{204-224}$ peptide forms hydrogen bonds to Arg297 and Asn376 of NO66 via the backbone carbonyl group and side chain.
Further hydrogen bonds are observed between Gln217 of the Rp18 ${ }^{204-224}$ peptide and Asn326 of NO66. The four C-terminal residues Ile219, Gly220, Lys221 and Pro222 of the Rpl8 ${ }^{204-224}$ peptide make van der Waals interactions with the side chains of Gln260, Leu299 and Tyr577 of NO66. In the active site of NO66, two histidine residues of the $\mathrm{Rp} 18^{204-224}$ peptide, His216 and His218, are accommodated in two deeply buried pockets. His218 forms a hydrogen bond to Ser421 and the distance between the $\mathrm{C}^{\beta}$ atom and the $\mathrm{Ni}^{2+}$ ion is $10 \AA$. His 216 is directed to the active centre by forming hydrogen bonds to Tyr328 and Ser421. The $\mathrm{C}^{\beta}$ atom of His216 is only $4.3 \AA$ away from the $\mathrm{Ni}^{2+}$ ion in the active site (Fig. $3 c$ ). The distance is close enough for $\mathrm{Fe}^{2+}$ in native NO66 to activate the coordinated $\mathrm{O}$ atom and catalyze the hydroxylation reaction. This observation is consistent with the results of biochemical analyses showing that NO66 hydroxylates the $\mathrm{C}^{\beta}$ atom of His216 (Ge et al., 2012). Alignment of the amino-acid residues involved in the interactions between NO66 and Rpl8 demonstrates that these residues are highly conserved in both proteins from different species, implying the importance of

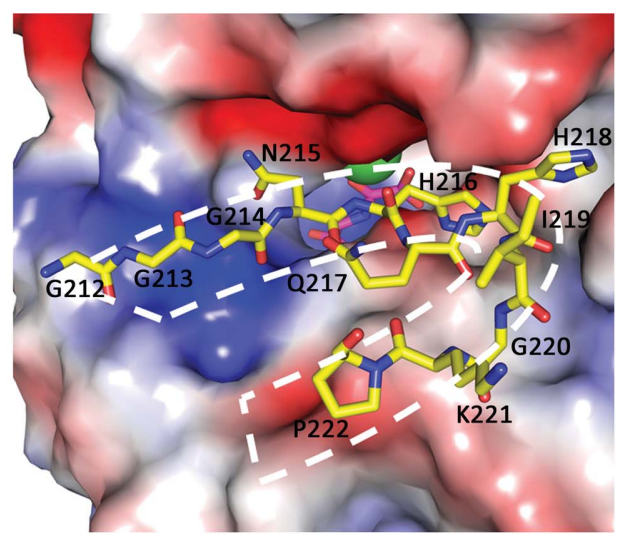

(a)

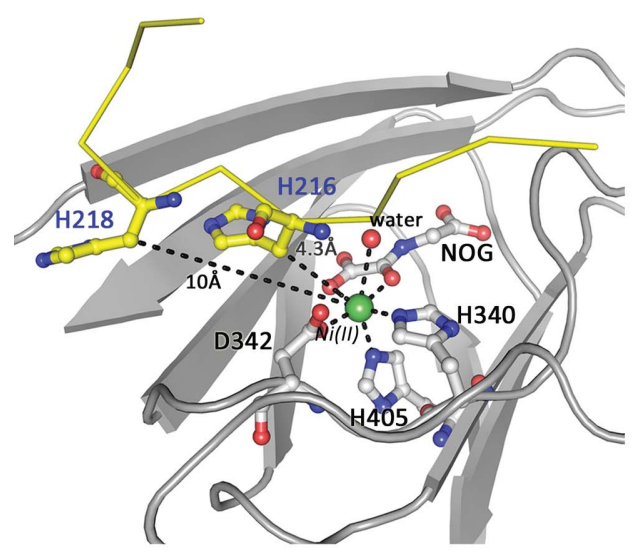

(c)

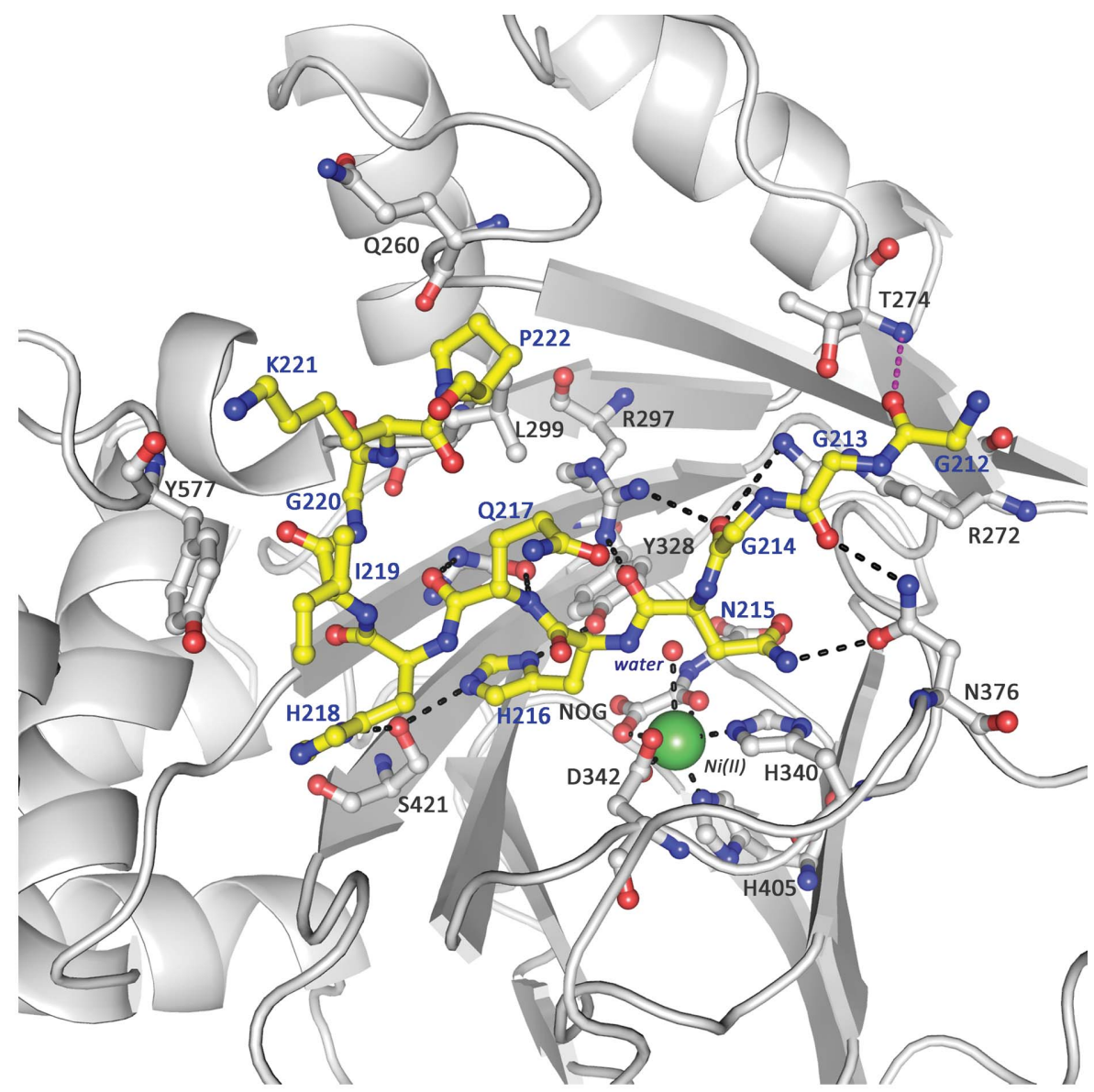

(b)

Figure 3

Specific recognition of Rpl8 by NO66. (a) A surface representation of the active site of NO66 is shown. Positively charged, negatively charged and neutral areas are shown in blue, red and white, respectively. The Rpl $8^{204-224}$ peptide adopts a U-shaped conformation in the active site of NO66. $(b)$ A detailed view of the interaction network of NO66 with Rpl $8^{204-224}$ and NOG. Rpl8 $8^{204-224}$ and the amino-acid residues of NO66 interacting with Rpl8 $8^{204-224}$ are shown as stick models. $\mathrm{Ni}^{2+}$ and water molecules are shown as spheres. NO66, $\mathrm{Rpl} 8^{204-224}, \mathrm{NOG}, \mathrm{Ni}^{2+}$ and water molecules are coloured grey, yellow, green, magenta and red, respectively. (c) His 216 is the hydroxylation site in Rpl8 catalyzed by NO66. The distances between the $\mathrm{C}^{\beta}$ atoms of His 216 and His 218 and the $\mathrm{Ni}^{2+}$ ion in the active site are shown. 
these interactions in anchoring Rpl8 to the active site of NO66 and in presenting the $\mathrm{C}^{\beta}$ atom of His216 in Rpl8 for hydroxylation (Fig. 4a, Supplementary Fig. S4).

\subsection{NO66 recognizes Rpl8 through a conserved motif}

Analysis of the complex structure of NO66 ${ }^{176-\mathrm{C}}$ and the $\mathrm{Rpl}^{204-224}$ peptide demonstrated that NO66 binds to Rpl8 via hydrogen bonds and van der Waals interactions. The side chains of Asn215, His216 and His218 form hydrogen bonds to amino-acid residues from NO66, which determines the specificity of the recognition between NO66 and Rpl8. The $\mathrm{N}$-terminal three glycine residues are highly flexible and provide an opportunity for Rpl 8 to fit into the shallow groove at the entrance of the active site of NO66. Amino-acid sequence alignment shows that the region of Rpl8 recognized by NO66 is highly conserved (Fig. 4a). Previous biochemical studies reported that the $\mathrm{NH} X \mathrm{H}$ motif is preferred for NO66 hydroxylation (Ge et al., 2012). Based on our structural analysis and the result of multiple sequence alignment (Fig. 3 and Supplementary Fig. S4), we confirmed that NO66 recognizes the $\mathrm{NH} X \mathrm{H}$ motif (where $X$ is any amino-acid residue) and the flexibility of three consecutive glycine residues helps Rpl8 enter the active site of NO66. For further investigation, we performed a series of mutation experiments in Rpl8 and examined their interactions with NO66 by pull-down assays. Compared with wild-type Rp18, mutating Asn215, His216 and His218 to alanine completely abolished the binding to NO66. In contrast, mutating Gln217 and Ile219 to alanine has only a

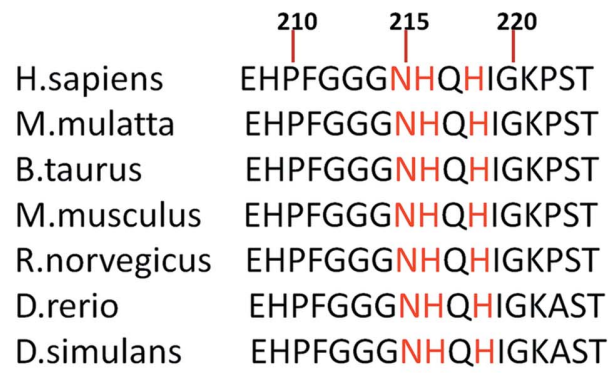

(a)

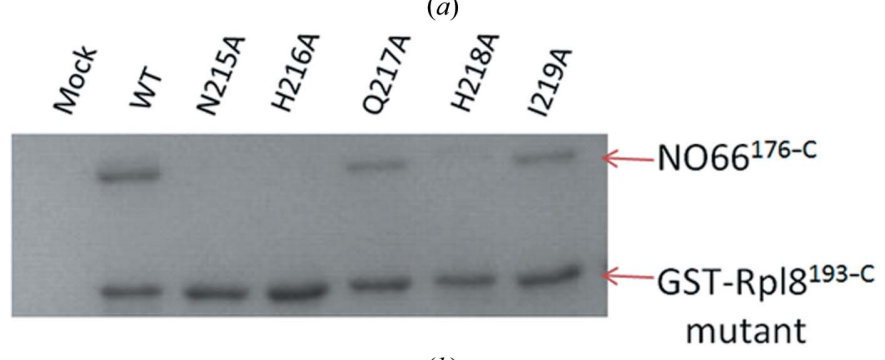

(b)

Figure 4

NO66 recognizes Rpl8 through a consensus motif. (a) A sequence alignment of the fragment in Rpl8 which is recognized by NO66 is shown. The accession numbers of the protein sequences are Homo sapiens, NP_150644 (human); Macaca mulatta, NP_001253030 (rhesus macaque); Bos taurus, NP_001029797 (cattle); Mus musculus, NP_036183 (mouse); Rattus norvegicus, NP_001030088 (rat); Danio rerio, NP_957007 (fish); Drosophila simulans, NP_728756 (fly). The residues that are critical for the binding of Rpl8 to NO66 are highlighted in red. (b) Pull-down of NO66 ${ }^{176-C}$ by wild-type Rpl $8^{193-C}$ and mutants of Rpl $8^{193-C}$ fused to GST are shown. slight effect on the interaction between the mutant proteins and NO66 (Fig. 4b). The pull-down assays results are consistent with our structural analysis.

3.5. Conformational changes of NO66 induced by substrate binding

Biochemical analysis showed that tetramerization is required for NO66 to catalyze hydroxylation efficiently. To better understand the mechanism, we compared the structure of the complex of NO66 ${ }^{176-\mathrm{C}}$ and $\mathrm{Rpl} 8^{204-224}$ with the structure of $\mathrm{NO}^{176-\mathrm{C}}-\mathrm{Ni}^{2+}$ (PDB entry $\left.4 \mathrm{e} 4 \mathrm{~h}\right)$. The overall structure of monomeric NO66 is very similar in both NO66 ${ }^{176-\mathrm{C}}$ complexed with $\mathrm{Rpl} 8^{204-224}$ and $\mathrm{NO}^{176-\mathrm{C}}-\mathrm{Ni}^{2+}$, with an r.m.s.d. of $0.28 \AA$ for all aligned amino-acid residues (Fig. $5 a$ ). Major conformational changes were observed at the active site. Upon substrate binding, $\beta \mathrm{X}$ (residues 271-274), $\beta \mathrm{Y}$ (residues 262268), $\beta Z$ (residues 404-408) and several loops shift towards the centre of the substrate-binding site owing to the interactions between NO66 and Rpl8 described previously (Fig. $3 b$ ). We further compared the tetrameric structure of the NO66 ${ }^{176-\mathrm{C}}-\mathrm{Ni}^{2+}$ and $\mathrm{NO}^{2176-\mathrm{C}}-\mathrm{Rp}^{204-224}$ complexes. In contrast to the similarity of the monomeric structure, the relative positions of the active sites of each subunit change in the two structures. The distance between the active sites in the dimer linked by interface II is $1.8 \AA$ closer in the complex structure than in $\mathrm{NO}^{176-\mathrm{C}}-\mathrm{Ni}^{2+}$. In contrast, the same distance in the dimer connected by interface $\mathrm{I}$ is almost unaltered and the observed difference is only $0.4 \AA$ (Fig. 5b).

Our biochemical assay results showed that the deletion of interface II resulted in a decrease in the catalytic activity of NO66 (Fig. 1b). To further understand the role of interface II in the regulation of the activity of NO66, we determined the structure of the mutant protein M2 lacking the interface. As predicted, the M2 protein forms a dimer with interface I. The overall structure of monomeric M2 protein is almost identical to wild-type NO66, with an r.m.s.d. of $0.37 \AA$ (Supplementary Fig. S5). Interestingly, when the dimer structure of the M2 protein was superimposed onto the dimer of NO66 coupled in the same way, we found that one of the M2 molecules in the dimer moves outwards compared with the wild-type protein. When one of the subunits of the M2 dimer was superposed onto NO66, the other subunit of the M2 dimer rotates about $16^{\circ}$ and shifts $4.7 \AA$ (Fig. $5 c$ ).

\section{Discussion}

NO66 was reported to be a dual-location protein found in both the nucleus and the nucleolus (Eilbracht et al., 2004). Previous studies showed that NO66 is a histone demethylase in vivo which is involved in gene-transcription regulation in the nucleus (Sinha et al., 2010; Brien et al., 2012; Sinha et al., 2014). However, recent studies demonstrated that NO66 has protein-hydroxylation activity and could control the biogenesis of ribosomes in the nucleolus (Ge et al., 2012). Because the function of NO66 remains unclear, we examined its activity in both histone demethylation and protein 


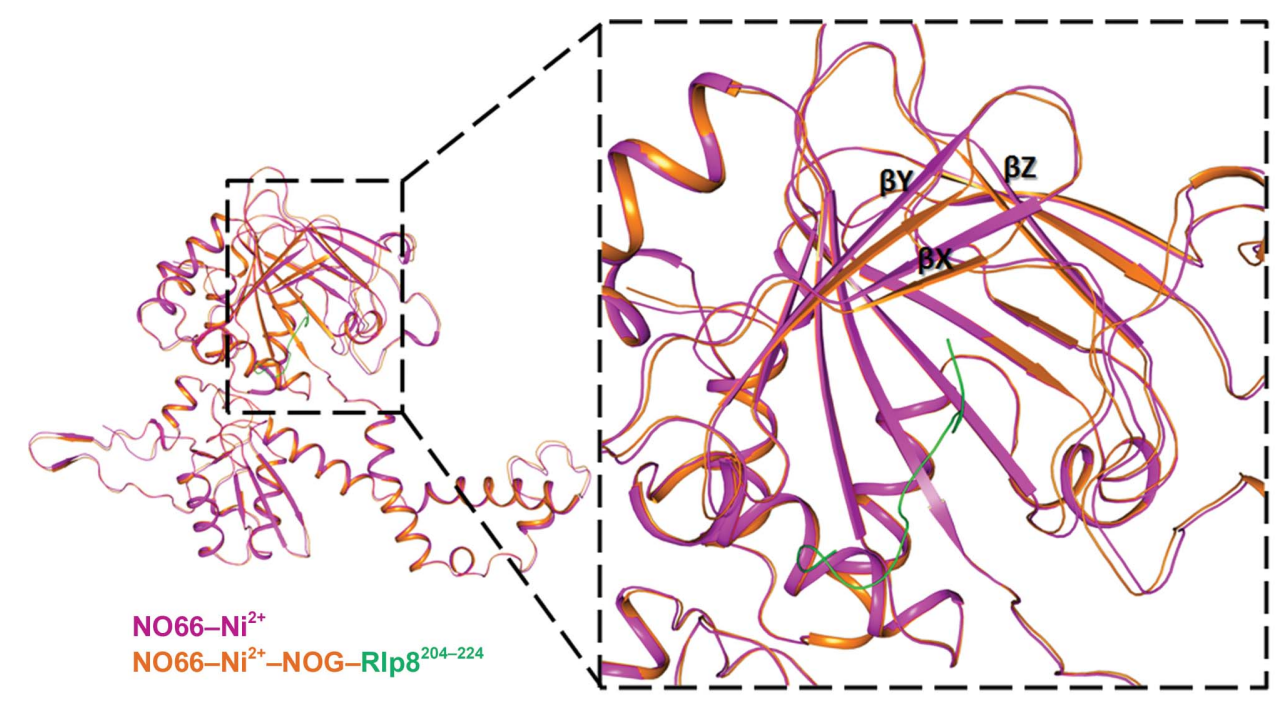

(a)
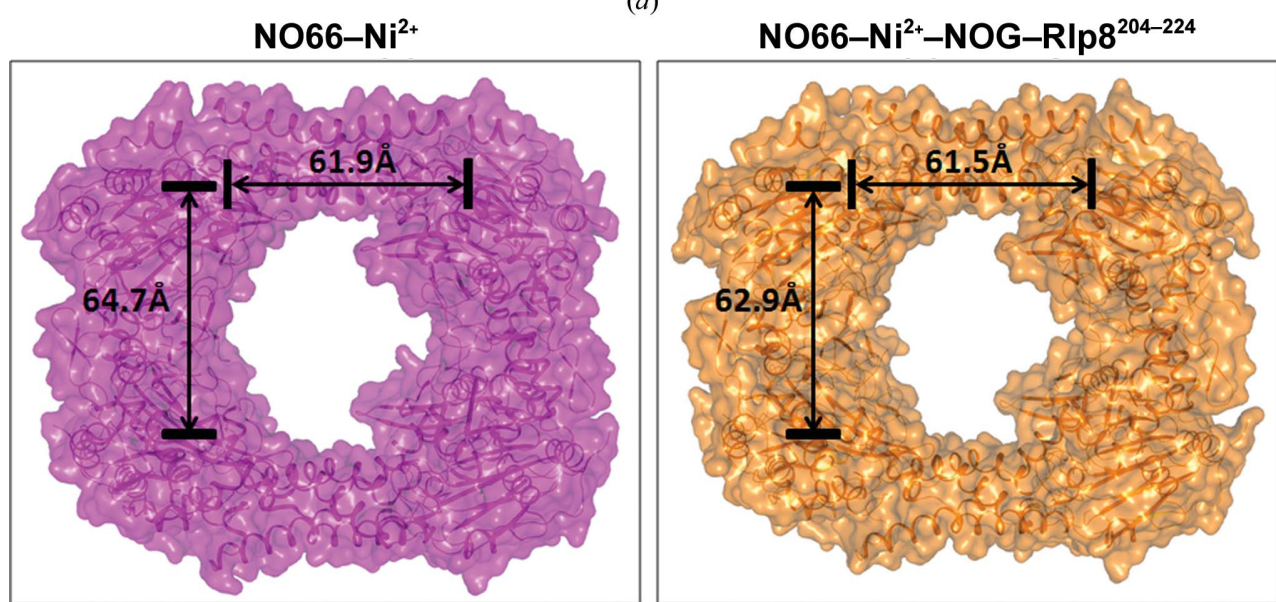

(b)

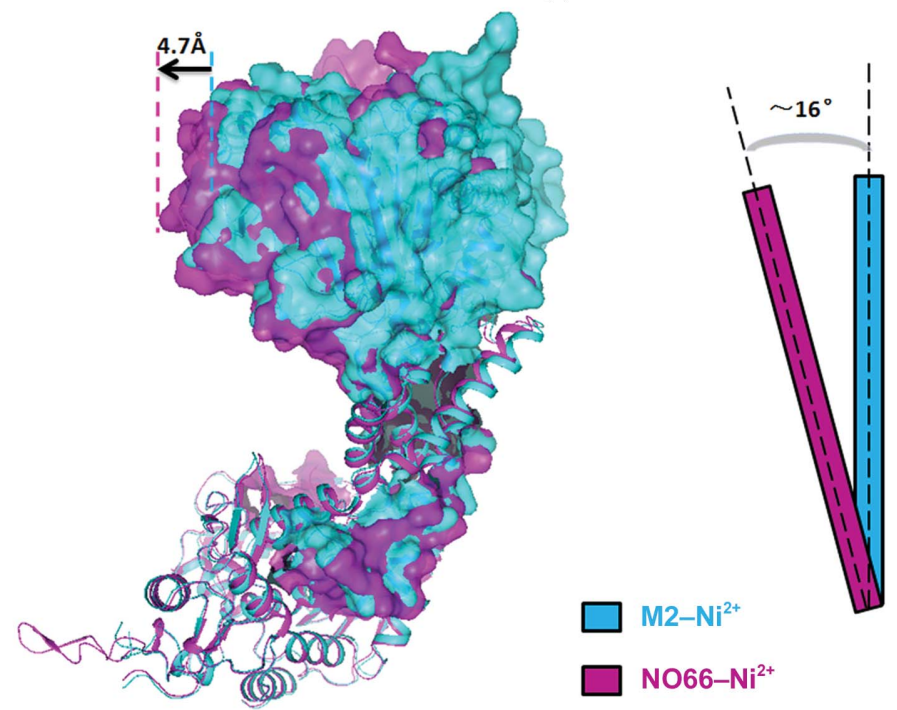

(c)

Figure 5

Conformational changes of NO66 are induced by substrate binding. (a) A comparison of the complex structure of $\mathrm{NO}^{176-\mathrm{C}}-\mathrm{Rpl}^{204-224}$ (orange) with $\mathrm{NO}^{176-\mathrm{C}}-\mathrm{Ni}^{2+}$ (magenta) is shown. The enlarged view shows the conformational changes in the active site. The three $\beta$-sheets $\beta \mathrm{X}(271-274), \beta \mathrm{Y}(262-268)$ and $\beta \mathrm{Z}$ (404-408) and several loops are rearranged upon substrate binding. (b) Surface representation of NO66 ${ }^{176-}$ $\mathrm{C}_{-} \mathrm{Ni}^{2+}$ (left) and the complex between NO66 ${ }^{176-\mathrm{C}}$ and $\mathrm{Rpl}^{204-224}$ (right) are shown. The distances between the active sites of NO66 in the two types of dimers are indicated. (c) A comparison of the structure of M2 (cyan) with one dimer of wild-type NO66 associated by interface I (magenta) is shown. hydroxylation. We did not observe the demethylation of histone peptides under the conditions usually used for such reactions in vitro. By contrast, hydroxylation activity of NO66 was clearly observed in our assays when a synthetic peptide derived from Rpl8 was used as a substrate (Supplementary Figs. S1a and $\mathrm{S} 1 b)$, which is consistent with previous findings (Ge et al., 2012; Chowdhury et al., 2014). However, the possibility could not be excluded that NO66 functions as a demethylase in vivo when assisted by other factors or under different conditions.

To gain insight into the molecular mechanisms for the enzymatic activity of NO66, we solved the crystal structure of NO66 ${ }^{176-C}$ in complex with $\mathrm{Rp} 18^{204-224}$ at $2.2 \AA$ resolution. In the complex structure, $\mathrm{Rpl} 8^{204-224}$ forms a U-shaped conformation with a bulge-shaped structure in the middle of the peptide. The $\mathrm{N}$ - and C-terminus of $\mathrm{Rp} 18^{204-224}$ are pushed close to each other and the middle of the peptide protrudes from the end (Fig. 3a). As a result, His 216 and His 218 locate in the middle of the Rpl8 ${ }^{204-224}$ peptide inserted into the binding pockets and His216 is directed to the active site for hydroxylation. As demonstrated by the complex structure, only the side chains of Asn215, His216 and His218 of Rpl8 form hydrogen bonds to NO66. Point mutations of this motif disrupt the interactions between NO66 and Rpl8 (Fig. 4b), which is consistent with the alanine-scanning results (Ge et al., 2012).

Our native substrate-bound structure of NO66 reveals a different conformation of the Rpl8 peptide compared with the Rpl8 peptides in the previously published cross-linked structures (Chowdhury et al., 2014), demonstrating the native interaction mechanism between NO66 and Rpl8 (Fig. 3). Comparison of 
Rpl8 peptides between our natural state structure and the cross-linked structures (Chowdhury et al., 2014) shows allatom r.m.s.d.s of $1.783 \AA$ for complex 1 (PDB entry $4 \mathrm{ccm}$ ), $1.622 \AA$ for complex 2 (PDB entry $4 \mathrm{ccm}$ ) and $2.152 \AA$ for complex 3 (PDB entry 4cco) (Fig. 6). The cross-linked structures show a much higher value of the average $B$ factor for the peptides for both the whole chain and the critical residues (Supplementary Table S1), indicating that cross-linked peptides do not stably interact with NO66.

The results obtained from our structural and biochemical analyses are in agreement with recently reported functional studies of NO66 in ribosome biogenesis (Eilbracht et al., 2004). However, NO66 has also been demonstrated to associate with chromatin and to regulate gene transcription (Sinha et al., 2010, 2014). The hydroxylation of Rpl8 is unable to accomplish this task, which suggested that NO66 may have additional substrates. A BLAST search identified approximately 20 proteins that contain sequences similar to the consensus motif recognized by NO66 (Supplementary Table S2). These proteins are potential substrates of NO66 and some of them are involved in the regulation of gene transcription. Consequently, we hypothesize that NO66 may have additional substrates in vivo and this could affect gene transcription indirectly.

From the results of our biochemical assays, we observed that NO66, when assembled in a tetrameric form, catalyzes the hydroxylation of Rpl8 most efficiently. Disruption of the tetrameric assembly in various ways leads to loss of catalytic activity (Fig. 1b). Oligomerization has been demonstrated to be an efficient way to control the activity of JmjC domaincontaining enzymes. For example, destabilization of the FIH dimer results in an enzymatically inactive monomer (Lancaster et al., 2004). In addition, disruption of dimerization in Mina53 and YcfD causes a loss of activity (Chowdhury et al., 2014). These biochemical results reveal that some $\mathrm{JmjC}$ domain-containing enzymes exist in oligomeric

Figure 6 forms, which might be important for control of their activity.

To further understand the mechanism, we determined the structure of the mutant protein $\mathrm{M} 2$ and compared the NO66 ${ }^{176-\mathrm{C}}-\mathrm{Rp} 18^{204-224}$ complex structure with the NO66- $\mathrm{Ni}^{2+}$ structure (PDB entry 4e4h) and the M2 structure. We found that in the tetrameric structure of NO66 $6^{176-\mathrm{C}}$ complexed with Rpl $8^{204-224}$ the active sites of NO66 in the dimer related by both interface I and interface II move closer to each other upon substrate binding (Fig. $5 b$ ). In addition, Mina53 and YcfD form dimers mediated by interfaces similar to interface I of NO66 (Chowdhury et al., 2014). Interestingly, we noticed that the distances of the active sites in the dimer of Mina53

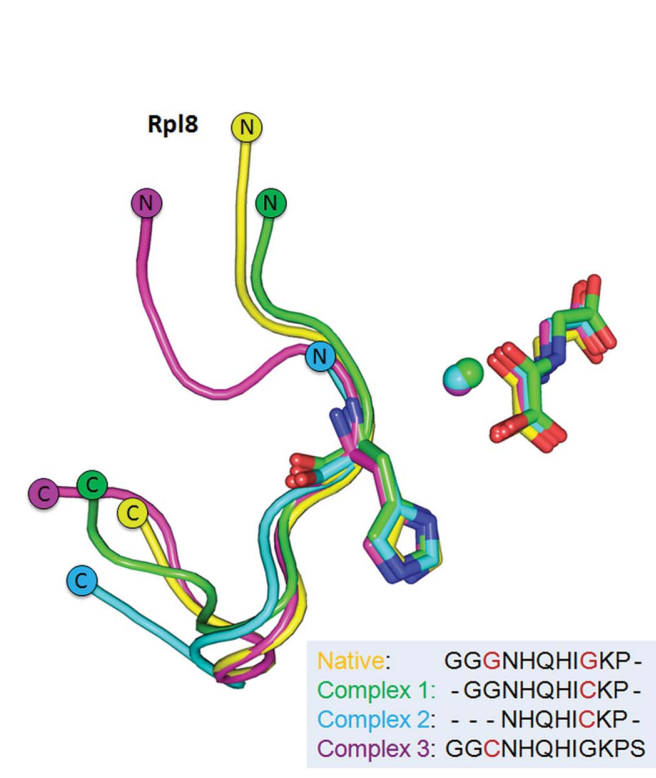

(a)

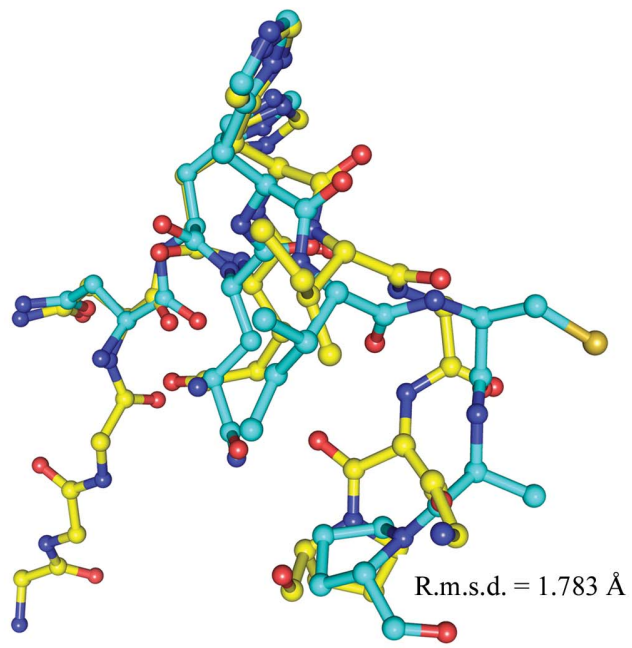

(c)

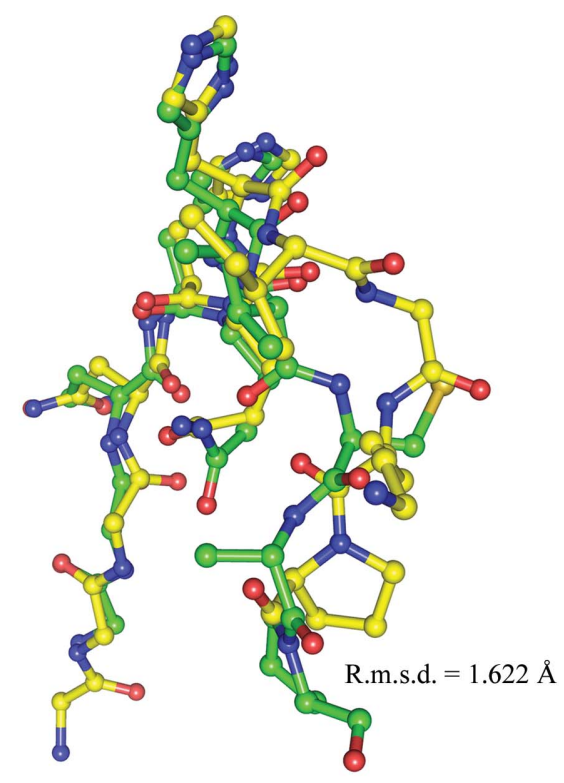

(b)

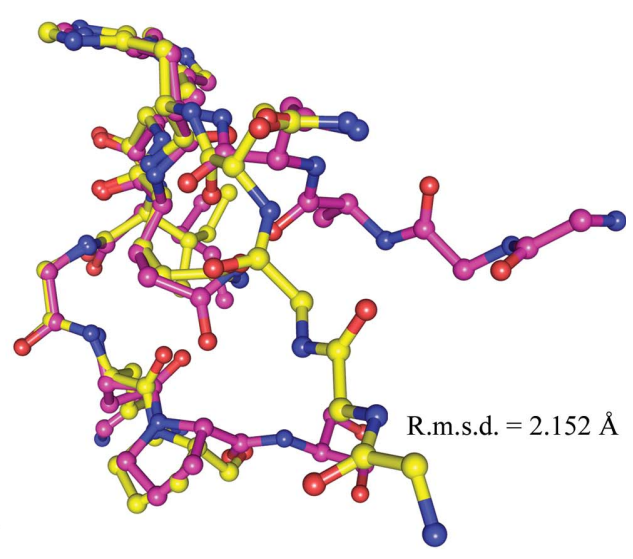

(d)

Comparison of structures of the Rpl8 peptide bound to NO66. (a) Chowdhury et al. (2014) used a crosslinking strategy to determine the complex structures of NO66 and Rpl8 peptides. In contrast, we determined the structure of NO66 ${ }^{176-\mathrm{C}}$ in complex with $\mathrm{Rp}^{204-224}$ in the native state. Cross-linking by disulfide bonds affects the conformation of the Rpl8 peptide bound to NO66. The Rpl8 peptide binds to NO66 in the native state and is coloured yellow. Rpl8 peptides cross-linked to NO66 are coloured green (complex 1; PDB entry 4ccm), blue (complex 2; PDB entry 4ccn) and magenta (complex 3; PDB entry $4 \mathrm{cco}$ ), respectively. Sequence-alignment results of Rpl8 peptides from these complex structures are shown at the bottom of the panel. Amino-acid residues mutated to cysteine are highlighted in red. $(b, c, d)$ Detailed comparison of the Rpl8 conformation between the native state and three cross-linked structures. 
(PDB entries 4bu2 and 4bxf) and YcfD (PDB entries 4csw and 4cug) were shortened in the same way when substrates were bound (Supplementary Fig. S6). In addition, the relative positions of the two subunits in the dimer of the mutant protein M2 shift outwards compared with wild-type NO66 (Fig. 5c). Interface II, which was deleted in M2, pulls these two subunits towards the other two subunits to form an NO66 tetramer. This tetramerization may further facilitate the shifting of the subunits when NO66 binds to its substrate. Although we were unable to determine how such conformational changes affected the activity of these enzymes, disruption of the oligomerization forms leads to a loss of catalytic activity (Fig. 1b). In this context, we proposed that oligomerization of NO66 might affect the motion of each subunit in the catalytic process and thus control the activity.

\section{Acknowledgements}

We thank the staff at beamline BL17U1 of the Shanghai Synchrotron Radiation Facility (SSRF) for assistance with data collection. This work was supported by the Strategic Priority Research Program of the Chinese Academy of Sciences (grant No. XDB 08010101). This work was also supported by grants from the Chinese Ministry of Science and Technology (No. 2012CB917202), the National Natural Science Foundation of China (Nos. 31370756, 31171241 and 31361163002) and a Scientific Research Grant from Hefei Science Center of CAS (No. 2015SRG-HSC043) to JZ.

\section{References}

Battye, T. G. G., Kontogiannis, L., Johnson, O., Powell, H. R. \& Leslie, A. G. W. (2011). Acta Cryst. D67, 271-281.

Brien, G. L. et al. (2012). Nature Struct. Mol. Biol. 19, 1273-1281.

Chen, Z., Zang, J., Whetstine, J., Hong, X., Davrazou, F., Kutateladze, T. G., Simpson, M., Mao, Q., Pan, C.-H., Dai, S., Hagman, J., Hansen, K., Shi, Y. \& Zhang, G. (2006). Cell, 125, 691-702.

Chowdhury, R., McDonough, M. A., Mecinović, J., Loenarz, C., Flashman, E., Hewitson, K. S., Domene, C. \& Schofield, C. J.
(2009). Structure, 17, 981-989.

Chowdhury, R. et al. (2014). Nature (London), 510, 422-426.

Clifton, I. J., McDonough, M. A., Ehrismann, D., Kershaw, N. J., Granatino, N. \& Schofield, C. J. (2006). J. Inorg. Biochem. 100, 644-669.

Eilbracht, J., Reichenzeller, M., Hergt, M., Schnölzer, M., Heid, H., Stöhr, M., Franke, W. W. \& Schmidt-Zachmann, M. S. (2004). Mol. Biol. Cell 15, 1816-1832.

Emsley, P. \& Cowtan, K. (2004). Acta Cryst. D60, 2126-2132.

Evans, P. (2006). Acta Cryst. D62, 72-82.

Ge, W. et al. (2012). Nature Chem. Biol. 8, 960-962.

Jenuwein, T. \& Allis, C. D. (2001). Science, 293, 1074-1080.

Kato, M., Araiso, Y., Noma, A., Nagao, A., Suzuki, T., Ishitani, R. \& Nureki, O. (2011). Nucleic Acids Res. 39, 1576-1585.

Lancaster, D. E., McNeill, L. A., McDonough, M. A., Aplin, R. T., Hewitson, K. S., Pugh, C. W., Ratcliffe, P. J. \& Schofield, C. J. (2004). Biochem. J. 383, 429-437.

Laskowski, R. A., MacArthur, M. W., Moss, D. S. \& Thornton, J. M. (1993). J. Appl. Cryst. 26, 283-291.

Martin, C. \& Zhang, Y. (2005). Nature Rev. Mol. Cell Biol. 6, 838-849.

McCoy, A. J., Grosse-Kunstleve, R. W., Adams, P. D., Winn, M. D., Storoni, L. C. \& Read, R. J. (2007). J. Appl. Cryst. 40, 658-674.

McDonough, M. A., Loenarz, C., Chowdhury, R., Clifton, I. J. \& Schofield, C. J. (2010). Curr. Opin. Struct. Biol. 20, 659-672.

Murshudov, G. N., Skubák, P., Lebedev, A. A., Pannu, N. S., Steiner, R. A., Nicholls, R. A., Winn, M. D., Long, F. \& Vagin, A. A. (2011). Acta Cryst. D67, 355-367.

Myllyharju, J. \& Kivirikko, K. I. (2004). Trends Genet. 20, 33-43.

Que, L. Jr (2000). Nature Struct. Biol. 7, 182-184.

Ruan, J., Ouyang, H., Amaya, M. F., Ravichandran, M., Loppnau, P., Min, J. \& Zang, J. (2012). PLoS One, 7, e35376.

Sinha, K. M., Yasuda, H., Coombes, M. M., Dent, S. Y. R. \& de Crombrugghe, B. (2010). EMBO J. 29, 68-79.

Sinha, K. M., Yasuda, H., Zhou, X. \& deCrombrugghe, B. (2014). J. Bone Miner. Res. 29, 855-865.

Tao, Y., Wu, M. H., Zhou, X., Yin, W., Hu, B., de Crombrugghe, B., Sinha, K. M. \& Zang, J. (2013). J. Biol. Chem. 288, 16430-16437.

Webby, C. J. et al. (2009). Science, 325, 90-93.

Whetstine, J. R., Nottke, A., Lan, F., Huarte, M., Smolikov, S., Chen, Z., Spooner, E., Li, E., Zhang, G., Colaiacovo, M. \& Shi, Y. (2006). Cell, 125, 467-481.

Winn, M. D. et al. (2011). Acta Cryst. D67, 235-242.

Zhou, X., Tao, Y., Wu, M., Zhang, D. \& Zang, J. (2012). Acta Cryst. F68, 764-766. 